\title{
Large Magnetoelectric Effects in Electrodeposited Nanoporous Microdisks Driven by Effective Surface Charging and Magneto-lonics
}

\author{
Cristina Navarro-Senent, ${ }^{* \dagger}{ }^{\dagger}$ Jordina Fornell, $^{\dagger}$ Eloy Isarain-Chávez, ${ }^{\dagger}$ Alberto Quintana ${ }^{\dagger}{ }^{\dagger}$ \\ Enric Menéndez, ${ }^{\dagger}$ Michael Foerster, Lucía Aballe, ${ }^{\ddagger}$ Eugen Weschke, ${ }^{\S}$ Josep Nogués, ${ }^{\|, \perp}$ Eva Pellicer, ${ }^{*},{ }^{\dagger}$ \\ and Jordi Sort $*,+, \perp_{(0)}$ \\ ${ }^{\dagger}$ Departament de Física, Universitat Autònoma de Barcelona, Cerdanyola del Vallès, E-08193 Barcelona, Spain \\ ${ }^{\ddagger}$ Alba Synchrotron Light Facility, CELLS, Cerdanyola del Vallès, E-08280 Barcelona, Spain \\ ${ }^{\S}$ Helmholtz-Zentrum Berlin für Materialien und Energie, Albert-Einstein-Strasse 15, D-12489 Berlin, Germany \\ "Catalan Institute of Nanoscience and Nanotechnology (ICN2), CSIC and The Barcelona Institute of Science and Technology, \\ Campus UAB, Bellaterra, E-08193 Barcelona, Spain \\ ${ }^{\perp}$ ICREA, Pg. Lluís Companys 23, E-08010 Barcelona, Spain
}

Supporting Information

ABSTRACT: A synergetic approach to enhance magnetoelectric effects (i.e., control of magnetism with voltage) and improve energy efficiency in magnetically actuated devices is presented. The investigated material consists of an ordered array of $\mathrm{Co}-\mathrm{Pt}$ microdisks, in which nanoporosity and partial oxidation are introduced during the synthetic procedure to synergetically boost the effects of electric field. The microdisks are grown by electrodeposition from an electrolyte containing an amphiphilic polymeric surfactant. The bath formulation is designed to favor the incorporation of oxygen in the form of cobalt oxide. A pronounced reduction of coercivity (88\%) and a remarkable increase of Kerr signal amplitude (60\%) are observed at room temperature upon subjecting the microdisks to negative voltages through an electrical double layer. These large voltage-induced changes in the magnetic properties of the microdisks are due to (i) the high surface-area-to-volume ratio with ultranarrow pore walls $(\mathrm{sub}-10 \mathrm{~nm}$ ) that promote enhanced electric charge accumulation and (ii) magneto-ionic effects, where voltage-driven $\mathrm{O}^{2-}$ migration promotes a partial reduction of $\mathrm{CoO}$ to $\mathrm{Co}$ at room temperature. This simple and versatile procedure to fabricate patterned "nano-in-micro" magnetic motifs with adjustable voltage-driven magnetic properties is very appealing for energy-efficient magnetic recording systems and other magnetoelectronic devices.

KEYWORDS: nanoporous material, magnetoelectric actuation, Co-Pt alloy, patterned microstructures, magneto-ionic effects

\section{INTRODUCTION}

The advent of spintronics has revolutionized information recording strategies and has triggered the development of a number of innovative devices with an ultrahigh data storage capacity operating at ultrafast processing rates. ${ }^{1-4}$ However, energy consumption continues to be an important bottleneck in these devices. Conventional magnetic systems utilize magnetic fields to write information. The electric currents required to create such magnetic fields involve a significant energy loss in the form of heat dissipation (Joule effect). One method to overcome the need for external magnetic fields is to use spin-polarized currents (spin-torque effect). ${ }^{4}$ However, generation of spin-polarized currents is still energetically costly. The use of an applied voltage rather than magnetic fields or spin-polarized currents would represent a significant breakthrough to decrease power consumption. ${ }^{5-10}$

Recent studies have reported on the possibility to tailor the magnetic properties of materials (e.g., magnetic anisotropy, magnetization, or coercivity) with electric fields. ${ }^{6-8}$ There are various ways to manipulate magnetism using voltage: (i) development of single-phase multiferroics, in which magnetic and electric orders are mutually coupled; ${ }^{9,11}$ (ii) strainmediated magnetoelectric coupling in piezoelectric/magnetostrictive composites, where the voltage-induced strain in the piezoelectric is transmitted to the ferromagnetic counterpart via inverse magnetostriction; ${ }^{10,12-14}$ (iii) carrier modulation effect, where the electronic band structure (and, hence, the resulting magnetism) of ultrathin ferromagnetic films ${ }^{15-21}$ or magnetic semiconductors ${ }^{22-24}$ is modified due to the electrostatic charge accumulation at the surface; and (iv) magnetoionics (i.e., ionic migration observed in some ferromagnetic metallic layers in direct contact with oxides such as $\mathrm{Gd}_{2} \mathrm{O}_{3}$ or

Received: October 6, 2018

Accepted: December 6, 2018

Published: December 6, 2018 
$\mathrm{HfO}_{2}$, which act as ion reservoirs by accepting or donating oxygen ions depending on the voltage polarity and strength $^{25-30}$ or $\mathrm{Fe}_{2} \mathrm{O}_{3}$ systems, which have been exploited in multilevel memristor devices ${ }^{31}$ ). Each of these strategies suffers from its own limitations: (i) single-phase multiferroics operative at room temperature are quite scarce; (ii) strain in piezoelectric/magnetostrictive thin films is reduced due to the clamping with the substrate, and fatigue effects would also limit the endurance of an eventual strain-mediated spintronic device; (iii) surface charge accumulation in metallic films is limited by the Thomas-Fermi electric-field screening length (on the order of $0.5 \mathrm{~nm}$ ), ${ }^{32}$ hence being only effective in ultrathin films; and (iv) magneto-ionics often requires thermal treatments (since ionic migration is thermally assisted) and it only occurs in some specific metal-metal oxide systems. ${ }^{25-30}$

In this work, a new strategy to boost the effect of voltage on the magnetic properties at room temperature based on the combination of surface charge accumulation and magnetoionic effects is reported. To this end, nanoporous $\mathrm{Co}-\mathrm{Pt}$ microdisks with partly oxidized Co are prepared by electrodeposition and subsequently actuated with voltage in liquid configuration using anhydrous propylene carbonate (PC). The electric field is generated through the formation of an electrical double layer (EDL). ${ }^{33}$ The induced nanoporosity allows the whole disks (not only the outer surface) to be magnetoelectrically active since although electric field is confined within the Thomas-Fermi screening length, the very narrow pore walls together with the use of a liquid dielectric (which can penetrate into the three-dimensional (3D) nanoporous architecture of the disks) promote a very effective accumulation of electrostatic charges in spite of the relatively large thickness of the Co-Pt microdisks (>300 nm). Remarkably, our previous works demonstrated that the coercivity of some nanoporous alloys (e.g., $\mathrm{Cu}-\mathrm{Ni}^{34}$ or $\mathrm{Fe}-\mathrm{Cu}^{35}$ ) can be considerably reduced with voltage (to ca. $32 \%$ ), which already represented a significant improvement compared to seminal works in FePt and FePd ultrathin films, where the reported changes in coercivity were only of around $4.5 \% .^{15}$ In these works, the very narrow thickness of the EDL created at the interface between the sample and the electrolyte (of around $0.5 \mathrm{~nm}$ ) is crucial since it allows the generation of very strong electric fields (on the order of tens of $\mathrm{MV} / \mathrm{cm}$ ) for moderate values of applied voltages (few voltage). High-density electron accumulation via EDL gating has been also demonstrated to be an effective way to enhance the ferromagnetic response of Codoped $\mathrm{TiO}_{2}$ epitaxial films. ${ }^{36}$

Besides high surface electric charging, our work also takes advantage of magneto-ionic phenomena. So far, most works in the literature have used solid configurations (i.e., metallic ferromagnetic films adjacent to $\mathrm{GdO}_{x}$ or $\mathrm{HfO}_{2}$ layers $)^{25-30}$ in which oxygen ion migration has resulted in interesting magnetoelectric effects. Nevertheless, polar organic solvents (hence involving the formation of EDL) have been also utilized to promote changes of the magnetic properties of some oxide materials through controlled ion migration. For example, the saturation magnetization in $\mathrm{CuFe}_{2} \mathrm{O}_{4}$ and $\mathrm{ZnFe}_{2} \mathrm{O}_{4}$ films was varied at room temperature by electrochemically driven $\mathrm{Li}$ ion exchange. ${ }^{37}$ Similar changes were observed in $\mathrm{Ni}-\mathrm{Co}$ with an adjacent $\mathrm{HfO}_{2}$ layer when voltage was applied through an EDL. 38

Inspired by the above approaches (i.e., the benefit of nanoporosity to enhance charge accumulation and the use of an EDL to induce magneto-ionic effects at room temperature in oxide materials), here we investigate the effects of voltage on the magnetic properties of nanoporous $\mathrm{Co}-\mathrm{Pt} / \mathrm{CoO}$ composite micropatterned structures. We have selected the Co-Pt system as the metallic counterpart for several reasons: (i) it constitutes the basis of some materials currently used in magnetic storage media (due to its high anisotropy and high coercivity, even with a limited amount of $\mathrm{Pt}$ in the alloy composition), ${ }^{39,40}$ (ii) magnetoelectric effects due to charge accumulation have been predicted in $\mathrm{Co}-\mathrm{Pt}$ by means of density functional calculations, ${ }^{20}$ (iii) it is known that $\mathrm{Co}-\mathrm{Pt}$ is prone to incorporate oxygen in its structure during electrodeposition under specific conditions, ${ }^{41-43}$ and (iv) the orbital moment of $\mathrm{Co}-\mathrm{Pt}$ and, thus, its anisotropy are known to be quite sensitive to the degree of oxidation. ${ }^{44}$ The study was performed on patterned microdisks prepared by optical lithography and micelle-assisted electrodeposition as a first step toward the miniaturization of magnetoelectric devices and to eventually promote a further change of coercivity with voltage due to the enhanced surface-area-to-volume ratio.

The synthetic method to prepare the nanoporous disks is based on combining micelle-assisted electrodeposition with optical lithography. Micelle-assisted electrodeposition uses block-copolymer micelles as a soft template ${ }^{45,46}$ for inducing nanoporosity during the growth of the alloy within the photolithographed areas (as depicted in Figure 1a). The electrolyte contains neither a $\mathrm{pH}$ buffering agent nor a complexing agent so as to favor the growth of nanocomposite layers consisting of a Co-rich alloy and Co oxides. In the absence of $\mathrm{pH}$ buffering substances, solution alkalinization at the cathode causes hydroxide/oxide precipitation. This way, oxygen can be introduced in the film in a metal oxide form. The designed composition and morphology lead to outstanding magnetoelectric/magneto-ionic effects: the coercivity $\left(H_{\mathrm{C}}\right)$ is reduced by $88 \%$ and the Kerr signal amplitude at saturation (designated as $A_{\text {Kerr }}$ ) increases by $60 \%$ by subjecting the nanoporous $\mathrm{Co}-\mathrm{Pt}$ disks to a negative voltage. The initial values of $H_{\mathrm{C}}$ and $A_{\mathrm{Kerr}}$ tend to be progressively recovered after the applied voltage is removed. Observation of these effects in lithographed structures (not only in continuous porous films, as in previous works ${ }^{34,35}$ ) paves the way toward the development of magneto-ionic voltage-controlled micrometer-sized devices.

\section{RESULTS AND DISCUSSION}

2.1. Morphology and Structure of the Electrodeposited Nanoporous Co-Pt/CoO Microdisks. Nanoporous $\mathrm{Co}-\mathrm{Pt}$ microdisks with $55 \pm 2 \mu \mathrm{m}$ in diameter and 309 $\pm 10 \mathrm{~nm}$ in thickness were synthesized by electrodeposition from a chloride electrolyte on photolithographed $\mathrm{Cu} / \mathrm{Ti} / \mathrm{Si}$ substrates (see the Experimental Section). A representative scanning electron microscopy (SEM) image of a couple of nanoporous $\mathrm{Co}-\mathrm{Pt}$ microdisks is shown in Figure $1 \mathrm{~b}$. When a single microdisk was imaged at a higher magnification, a morphology consisting of acicular grains with a large amount of small pores between them (i.e., mesoporosity) was observed on top (Figure 1c). This acicular morphology has often been reported in dense electrodeposited Co-based alloys $(\mathrm{Co}-\mathrm{Ni}$, Co-Mo, Co-Pt). ${ }^{47,48}$ Interestingly, here, the needlelike morphology remains in spite of the perturbation caused by the micelles during cations discharge and electrocrystallization. Further structural analysis was carried out by transmission electron microscopy (TEM), where the cross section of the $\mathrm{Co}-\mathrm{Pt}$ microdisks reveals the occurrence of tiny nanopores of 


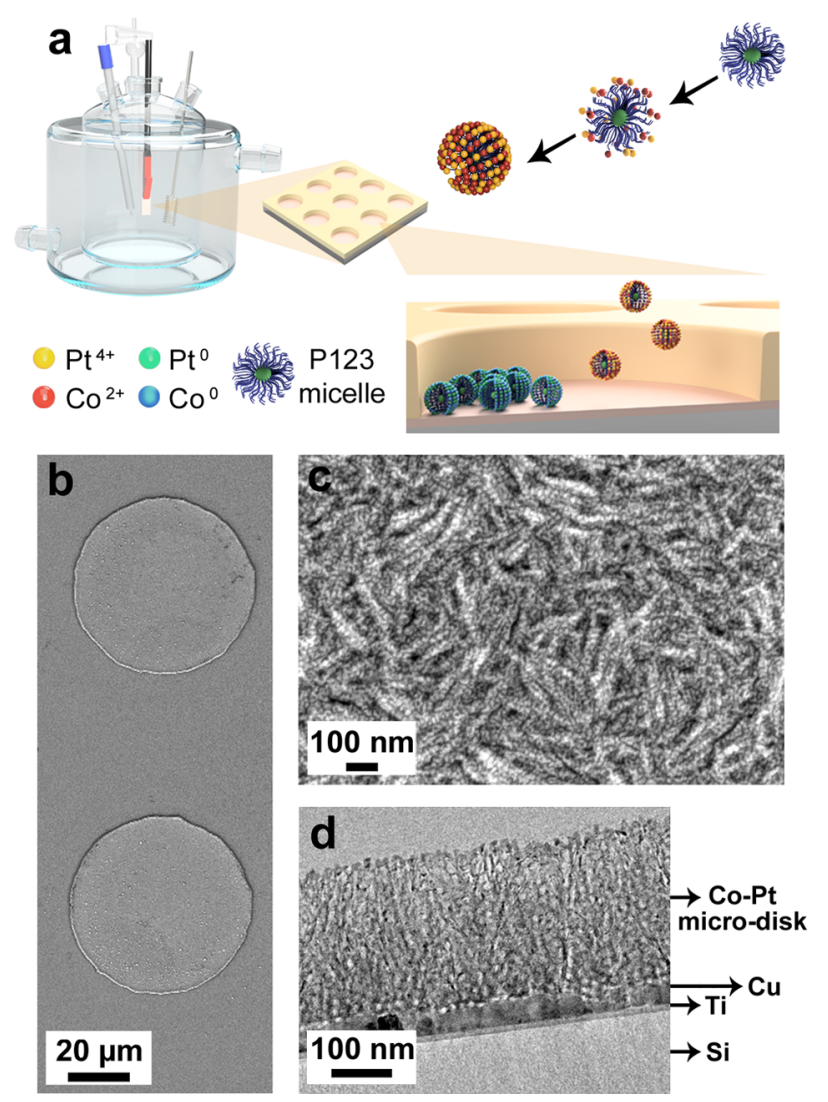

Figure 1. (a) Scheme of the micelle-assisted electrodeposition of Co$\mathrm{Pt} / \mathrm{CoO}$ on photolithographed substrates in a three-electrode electrochemical cell, wherein the dissolved metal species coordinate to the hydrophilic shell domains of the Pluronic P-123 triblock micelles; the metallic cations reduce in the conductive voids of the patterned substrate. (b) Scanning electron microscopy (SEM) image of two nanoporous Co-Pt microdisks. (c) High-resolution SEM image of a microdisk surface. (d) Cross-section transmission electron microscopy (TEM) image of a microdisk.

less than $10 \mathrm{~nm}$ in diameter all along the thickness of the disks, confirming that nanoporosity fully develops from the bottom to the top of the microdisks (Figure 1d). A columnar-like growth was also apparent from magnified cross-section TEM images (Figure 2a). Compositional analyses of the microdisks by energy-dispersive X-ray (EDX) spectroscopy (see the Supporting Information, Figure S1) revealed an average composition of $\mathrm{Co}_{70} \mathrm{Pt}_{30}$ (in at. \%) when only the metallic fraction of the deposit was considered. Furthermore, a significant amount of oxygen (20 at. \%) was also detected by EDX, thus confirming the presence of metal oxides in the motifs. The elemental atomic percentages in the microdisks are shown in Table S1 of the Supporting Information. Highresolution transmission electron microscopy (HRTEM) also revealed that the microdisks are nanocrystalline. Lattice fringes were observed in clusters of around 5-10 nm (Figure 2b), which correspond to the thickness of the pore walls. According to selected area electron diffraction (SAED) analysis (Figure $2 \mathrm{c}$ ), the nanoporous $\mathrm{Co}-\mathrm{Pt}$ microdisks consist mainly of a mixture of three phases: (i) hexagonal closed-packed (hcp) Co (space group $P_{63} / m m c$ ), (ii) cubic $\mathrm{CoO}$ phase (space group $\mathrm{F} m \overline{3} m$ ), and (iii) intermetallic $\mathrm{Co}_{3} \mathrm{Pt}$ (space group $\mathrm{Fm} \overline{3} m$ ). Note that the interplanar distances $\left(d_{h k l}\right)$ of the rings assigned to the hcp-Co phase were slightly shifted toward higher values with respect to those of pure hcp-Co, suggesting that a fraction of $\mathrm{Pt}$ was dissolved in the hcp-Co lattice.

2.2. Magnetoelectric Measurements. The effect of an externally applied electric field on the magnetic properties of the nanoporous $\mathrm{Co}-\mathrm{Pt} / \mathrm{CoO}$ microdisks was investigated by subjecting the sample to different constant voltages and measuring the hysteresis loops by magneto-optic Kerr effect (MOKE). As depicted schematically in Figure 3a, the sample was placed in an electrolytic cell, which contained a Pt wire as the counter electrode and anhydrous propylene carbonate (PC) with $\mathrm{Na}^{+}$and $\mathrm{OH}^{-}$ions as the electrolyte. Anhydrous PC is liquid enough at room temperature to properly penetrate into the pores and wet the whole sample and is broadly used not only in battery applications 49,50 but also in magnetoelectrically actuated systems. ${ }^{15,34,35,51,52} \mathrm{Na}^{+}$and $\mathrm{OH}^{-}$ions were formed after making residual water in the PC react with metallic Na. This procedure was aimed at: (i) removing any traces of water to minimize uncontrolled oxidation of the Co-

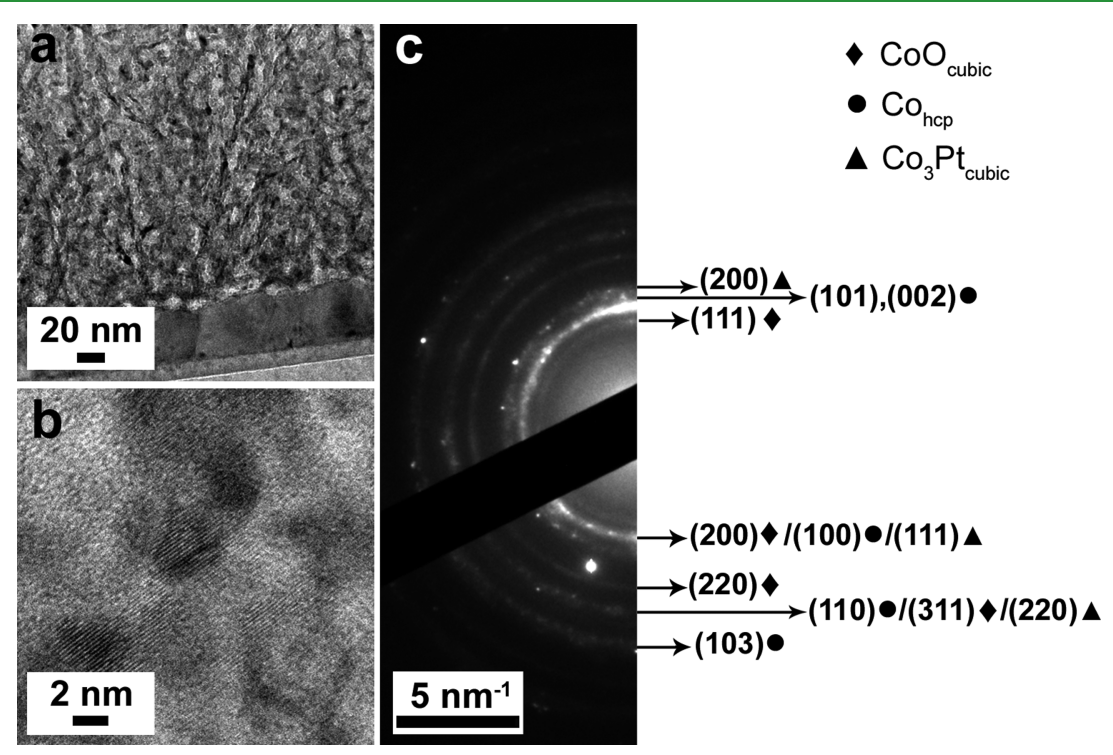

Figure 2. (a) TEM and (b) HRTEM images of the cross section of a nanoporous Co-Pt microdisk. (c) Corresponding SAED pattern. 

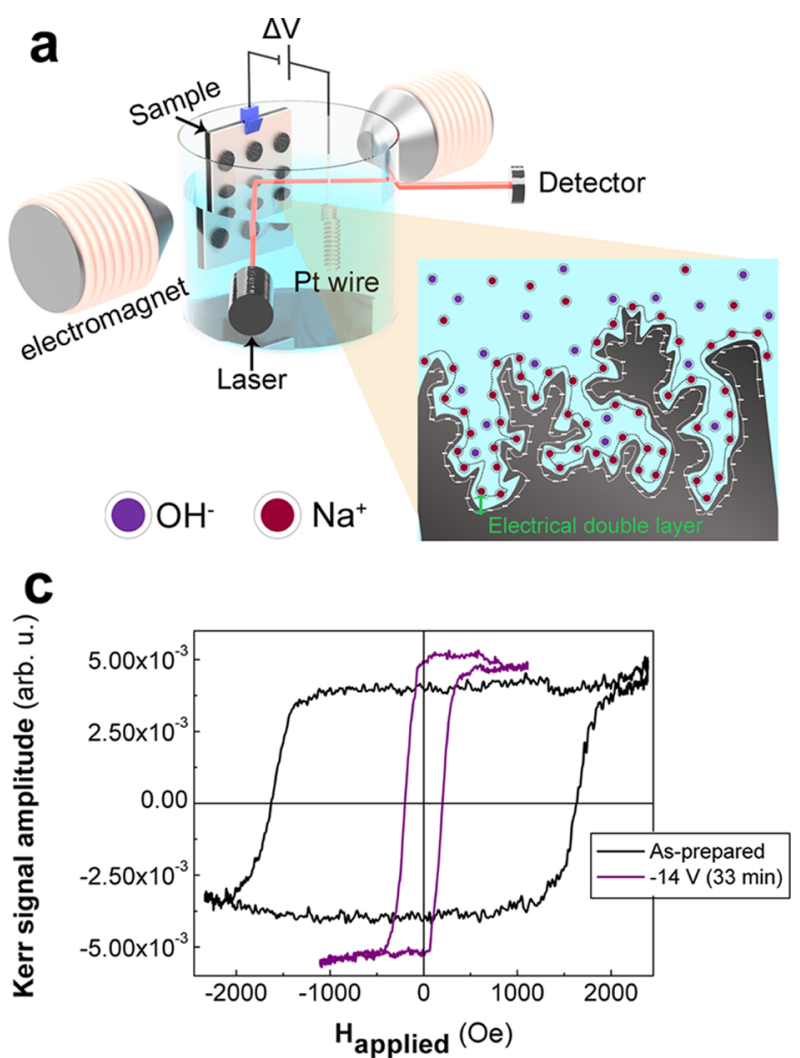
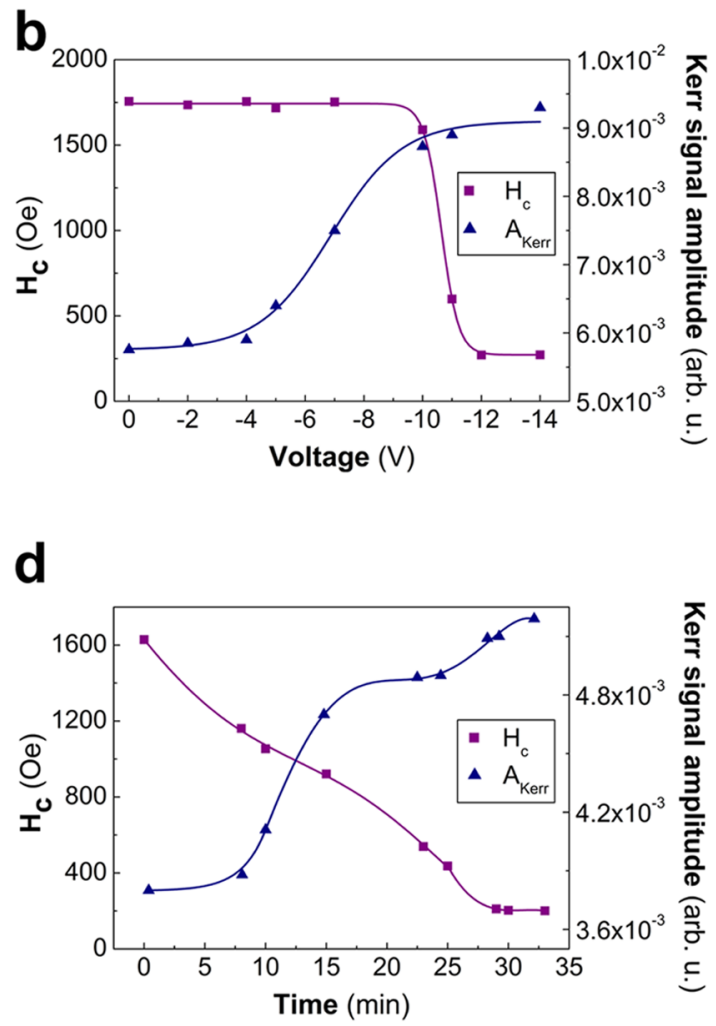

Figure 3. (a) Schematic drawing of the experimental setup used for the magnetoelectric measurements together with an illustration of the formation of the electrical double layer (EDL) at the interface between the nanopores and the electrolyte when the sample is subjected to a negative voltage adapted from ref 34. (b) Dependence of the coercivity $\left(H_{\mathrm{C}}\right)$ and Kerr amplitude signal at saturation (designated as $\left.A_{\text {Kerr }}\right)$ on the applied negative voltage for a fixed time of $10 \mathrm{~min}$ at each voltage value. (c) Representative hysteresis loops of the nanoporous $\mathrm{Co}-\mathrm{Pt} / \mathrm{CoO}$ microdisks measured at $0 \mathrm{~V}$ (black loop) and after applying $-14 \mathrm{~V}$ for $33 \mathrm{~min}$ (purple loop). (d) Dependence of $H_{\mathrm{C}}$ and $A_{\text {Kerr }}$ on the time during which a fixed voltage $-14 \mathrm{~V}$ was applied. The lines in panels $(\mathrm{b})$ and $(\mathrm{d})$ are guides to the eye.

Pt alloy and (ii) promoting the formation of the EDL, thus enhancing the magnitude of the electric field at the nanopore wall-electrolyte interface (Figure 3a). A current vs voltage profile compatible with the charge and discharge of the electric double layer was observed in the cyclic voltammetry curves taken in the electrolyte used to investigate microdisks' performance (Figure S4 of the Supporting Information).

First, hysteresis loops were recorded by focusing the laser spot onto a nanoporous $\mathrm{Co}-\mathrm{Pt}$ disk, applying different negative voltages ranging from 0 to $-14 \mathrm{~V}$ after waiting for $10 \mathrm{~min}$ at each voltage value. The dependence of $H_{\mathrm{C}}$ and $A_{\text {Kerr }}$ on the applied voltage is shown in Figure $3 \mathrm{~b}$ (the corresponding hysteresis loops are plotted in Figure S2 of the Supporting Information). Note that the Kerr signal amplitude is, in a first approximation, proportional to the magnetic moment. Substantial effects were observed after overcoming threshold voltages of -5 and $-10 \mathrm{~V}$ for $A_{\text {Kerr }}$ and $H_{\mathrm{C}}$, respectively. Remarkably, $H_{\mathrm{C}}$ was reduced by $85 \%$ (from 1756 to $271 \mathrm{Oe}$ ), whereas $A_{\text {Kerr }}$ increased by $60 \%$ with respect to its initial value. A time effect must be also considered since the time was accumulative due to the stepwise increase of voltage.

In a second series of measurements, carried out on a different sample, voltage was fixed at $-14 \mathrm{~V}$, and hysteresis loops were recorded varying the amount of time during which the voltage was applied (Figures 3c and S2 in the Supporting Information). Clear narrowing of the hysteresis loops was again observed after a few minutes, yielding a maximum relative variation of $88 \%$ in $H_{\mathrm{C}}$ (from 1629 to $202 \mathrm{Oe}$ ) and a relative change of $30 \%$ in $A_{\text {Kerr }}$ after $33 \mathrm{~min}$ (Figure $3 \mathrm{~d}$ ). Note that the relative variations in $H_{\mathrm{C}}$ and $A_{\text {Kerr }}$ tend to level off after $27 \mathrm{~min}$.

Next, the recovery process was investigated while keeping the sample immersed in the electrolyte. After having applied $-14 \mathrm{~V}$ for $33 \mathrm{~min}$, voltage was withdrawn, and after $3 \mathrm{~h}$ at $0 \mathrm{~V}$, $H_{\mathrm{C}}$ increased by $109 \%$ (from 202 to $424 \mathrm{Oe}$, recovering up to $26 \%$ of the initial value), whereas $A_{\text {Kerr }}$ decreased, tending to approach the initial value at $0 \mathrm{~V}$. After 1 day at $0 \mathrm{~V}, H_{\mathrm{C}}$ increased up to $607 \mathrm{Oe}$, showing a recovery of $37 \%$ of the initial value (see the Supporting Information, Figure S3). A further increase of $H_{\mathrm{C}}$ was obtained after long-term waiting at $0 \mathrm{~V}\left(H_{\mathrm{C}}=1400 \mathrm{Oe}\right.$ after 1 month), evidencing the slow dynamics of the recovery process at $0 \mathrm{~V}$. The observed nontotal recovery at $0 \mathrm{~V}$ proves that the mechanism responsible for the observed magnetic changes involves some structural changes in the sample, and the induced effects can only be erased by applying positive voltages. Faster reversibility in the $H_{\mathrm{C}}$ and $A_{\text {Kerr }}$ trends was attained by applying positive voltages. The coercivity increased up to $772 \mathrm{Oe}$ applying $+2 \mathrm{~V}$ for $10 \mathrm{~min}$, and $H_{\mathrm{C}}=860$ Oe was measured after applying +10 $\mathrm{V}$ for $10 \mathrm{~min}$ (recovering $52 \%$ of the initial value). Unfortunately, degradation and polymerization of the propylene carbonate at the surface of the porous alloy were observed for voltages above $+10 \mathrm{~V}$, hence hampering further analysis at positive voltages by MOKE due to lack of reflectivity. It is important to emphasize that similar variations 
a

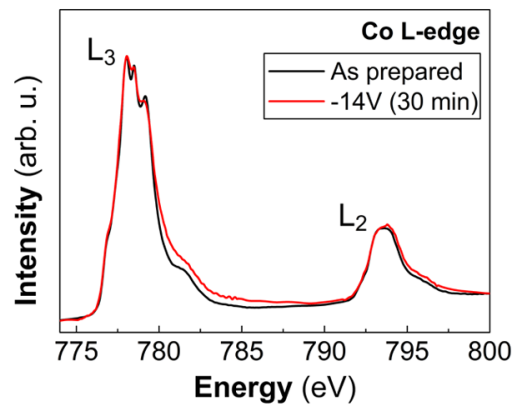

b

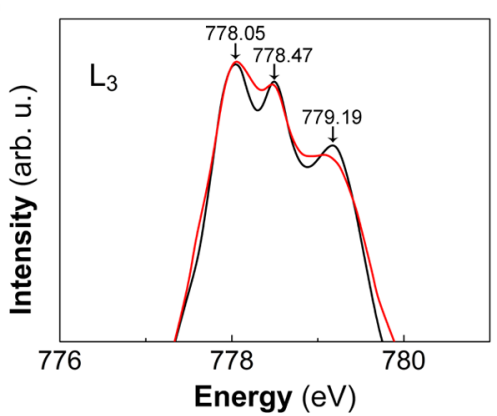

C

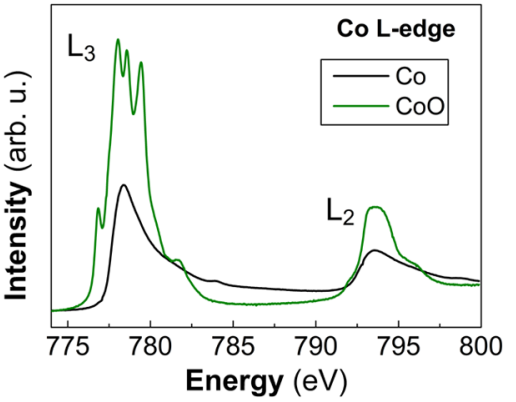

d

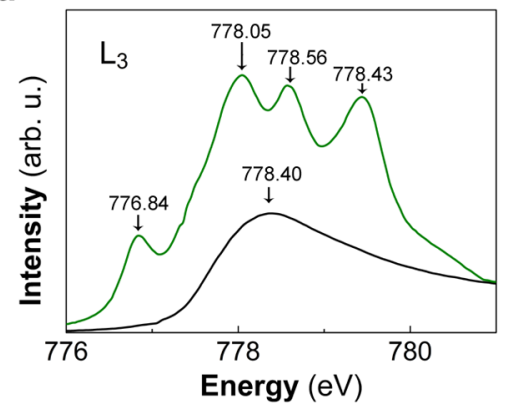

Figure 4. (a) Cobalt L-edge X-ray absorption spectra (XAS) of the nanoporous Co-Pt microdisks before (black curve) and after (red curve) applying $-14 \mathrm{~V}$ for $10 \mathrm{~min}$. (b) Zoom-in of the cobalt $\mathrm{L}_{3}$-edge where the energy position of the main peaks is indicated. (c) Cobalt $\mathrm{L}_{2,3}$-edges XAS spectra for references samples (in black: $20 \mathrm{~nm}$-thick Co thin, capped with $2 \mathrm{~nm}$ of Ta, grown by molecular beam epitaxy on top of a $\langle 100\rangle$ oriented $\mathrm{MgO}$ single crystal; in green: $\mathrm{CoO}$ (cobalt(II) oxide, 95\%) powders from Alfa Aesar). (d) Zoom-in of the cobalt $\mathrm{L}_{3}$-edge for the two reference samples with the corresponding energies of the main peaks indicated.

on the magnetic properties with voltage were obtained by performing the same magnetoelectric measurements on the same sample several months later, demonstrating the long lifetime of the nanoporous $\mathrm{Co}-\mathrm{Pt}$ microdisks.

Given the nanoporous nature of the investigated material, one plausible physical origin for the observed changes in $H_{\mathrm{C}}$ could be eventual variations in the magnetic anisotropy energy (MAE) due to the effective electric charge accumulation at the surface of the pore walls. In fact, density functional theory calculations for the $\mathrm{Co}-\mathrm{Pt}$ system have predicted a linear dependence of the MAE on the electric field, ${ }^{6,20,53}$ which could lead to variations in $H_{\mathrm{C}}$. However, there are several aspects that indicate that the effects observed here cannot be simply ascribed to changes in the electronic band structure of $\mathrm{Co}-\mathrm{Pt}$ induced by voltage. First, according to the theory, the response of the magnetocrystalline anisotropy (therefore $H_{C}$ ) to an applied voltage should be rather instantaneous and fully reversible (i.e., the dynamics of the system should be much faster than what is observed). Second, no significant changes in $A_{\text {Kerr }}$ are to be expected due to changes in the MAE. Third, our previous works on voltage-actuated nanoporous $\mathrm{Cu}-\mathrm{Ni}$ and $\mathrm{Fe}-\mathrm{Cu}$ alloys (with minimized oxygen content) led to relative variations in $H_{\mathrm{C}}$ of at most $32 \% .^{34,35}$ Finally, important variations in $A_{\text {Kerr }}$ occur already at lower applied voltages $(-5$ V) than the changes in $H_{\mathrm{C}}$, which start to be visible at $-10 \mathrm{~V}$ (see Figure $3 \mathrm{~b}$ ). Thus, an additional process is likely to dominate over the voltage-driven modification of the MAE related to the surface electric field. Given the presence of large amounts of oxygen in the as-prepared deposits, pronounced $\mathrm{O}^{2-}$ migration (magneto-ionic effect) ${ }^{25-30,43}$ is probably induced by voltage. The interplay between several additional possible magnetoelectric mechanisms in oxide materials immersed in electrolytes (e.g., $\gamma-\mathrm{Fe}_{2} \mathrm{O}_{3}$ ) has been discussed by several authors. ${ }^{54}$ Although redox reactions due to the use of aqueous electrolytes should be essentially ruled out in the present study, some minor effect stemming from elastic strain on the magnetic properties (due to the alteration of the atomic bonding caused by the charge accumulation) could still be present in our case, given the nanoporous nature of the investigated sample. Nevertheless, although ab initio studies predict variations in the magnetic properties around several percents due to magnetoelastic effects, ${ }^{55}$ the reported experimental changes so far have been between 0.5 and $3 \%$, much lower than in our results. ${ }^{56,57}$ This, together with the indepth structural characterization reported in the following sections, indicates that magneto-ionics is the most prominent mechanism responsible for the large magnetoelectric effects observed in our system.

2.3. Soft X-ray Absorption Characterization. To demonstrate the magneto-ionic origin of the obtained results, further chemical characterization by X-ray absorption spectroscopy (XAS) spectromicroscopy was performed on a single microdisk at the CIRCE beamline of the ALBA Synchrotron at the Co $\mathrm{L}_{2,3}$ edges (Figure 4 ). The XAS spectrum before voltage application exhibits a multiplet structure at the $\mathrm{L}_{3}$ edge and an asymmetric contribution at the $\mathrm{L}_{2}$ edge. This pattern is basically consistent with the $\mathrm{CoO}$ reference $\mathrm{XAS}^{58}$ (see Figure 4c) although the relative intensity of the various peaks in the $\mathrm{L}_{3}$ multipeak structure is not the same in the $\mathrm{Co}-\mathrm{Pt} / \mathrm{CoO}$ nanoporous disks and the $\mathrm{CoO}$ reference sample. This, together with the absence of a prominent $\mathrm{L}_{3}$ prepeak at around $776 \mathrm{eV}$ (which is a fingerprint of pure $\mathrm{CoO}$ ), suggests the coexistence of $\mathrm{CoO}$ with metallic $\mathrm{Co}$, in agreement with the SAED characterization, where both metallic and oxide phases were encountered (Figure 2c). 
After applying $-14 \mathrm{~V}$ for $40 \mathrm{~min}$ and removing the sample from the electrolyte (since XAS was performed ex situ, $30 \mathrm{~min}$ elapsed before starting the XAS measurements), the multipeak structure at the $\mathrm{Co}_{3}$ edge tends to smear out compared to the untreated sample (see Figure $4 \mathrm{~b}$ ). This suggests that after negative voltages, the amount of metallic Co increases at the expense of the $\mathrm{CoO}$ (note that metallic Co does not show the multipeak structure, Figure $4 \mathrm{c}, \mathrm{d}){ }^{58}$ The partial reduction from $\mathrm{CoO}$ to $\mathrm{Co}$ is consistent with the increase of the Kerr amplitude signal shown in Figure 3 since nanostructured $\mathrm{CoO}$ is likely paramagnetic at room temperature (note that bulk CoO has a Néel temperature of around $291 \mathrm{~K}) .^{59,60}$

2.4. Electron Energy Loss Spectroscopy (EELS) Characterization. Further characterization by electron energy loss spectroscopy (EELS) was employed to investigate the voltage-driven modifications of the spatial distribution of cobalt and oxygen in the Co-Pt microdisks at a local length scale. For this, a lamella was prepared by focused ion beam (FIB) and treated with voltage (see the Experimental Section). A scanning transmission electron microscopy (STEM) image of a cross-section of the film is shown in Figure 5a,b together

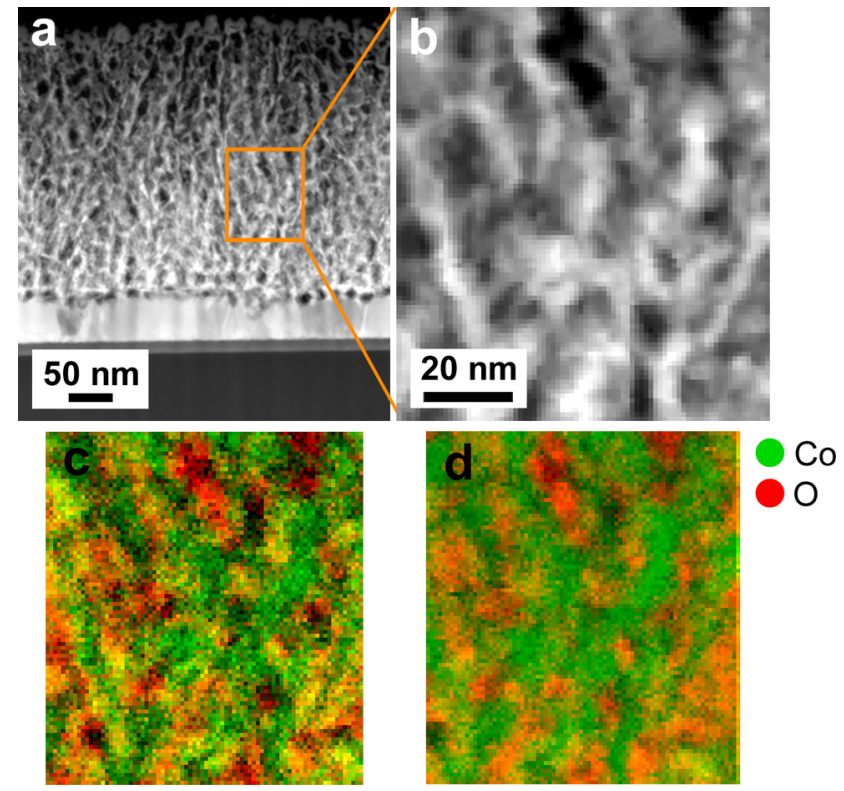

Figure 5. (a) STEM image of a cross section of a nanoporous $\mathrm{Co}-\mathrm{Pt}$ microdisk and (b) zoomed detail of the region enclosed in the orange box. The corresponding cobalt (green) and oxygen (red) EELS mappings are shown in (c) for the as-prepared sample and (d) after applying $-14 \mathrm{~V}$ for $40 \mathrm{~min}$.

with the corresponding Co (green) and O (red) EELS mappings in the as-prepared state (Figure 5c) and after applying $-14 \mathrm{~V}$ (Figure $5 \mathrm{~d}$ ). In the as-prepared sample, green regions are less defined and often superimposed to red pixels (rendering a yellowish color). The green regions are metallic Co, whereas the yellowish-like regions (superposition of green and red) correspond to $\mathrm{CoO}$. Note that there are some red areas as well (without being superimposed to green), which correspond to the pores filled with tetraethyl orthosilicate (TEOS), which was used during the STEM lamella preparation (see the Experimental Section). After the microdisk is subjected to $-14 \mathrm{~V}$, Co-rich regions become more clearly visible and tend to expand due to the voltage-driven $\mathrm{O}$ and Co redistribution, which results in enlarged Co metallic regions for negative voltages, in agreement with the XAS results (Figure 4).

The partial reduction from $\mathrm{CoO}$ to metallic Co can explain the decrease of coercivity. First, it is unlikely that the newly formed Co (after application of negative voltages) becomes readily alloyed with $\mathrm{Pt}$, and pure Co is known to typically exhibit lower $H_{\mathrm{C}}$ than hcp Co-Pt (although $H_{\mathrm{C}}$ is not an intrinsic parameter and is, of course, also dependent on the actual microstructure of the investigated material). Additionally, since the Co-rich magnetic regions increase in size during the voltage treatment (eventually coalescence can occur, as indicated in Figure 6), this also induces a decrease of the

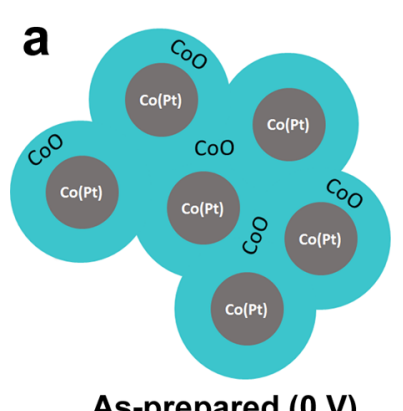

As-prepared (0 V)

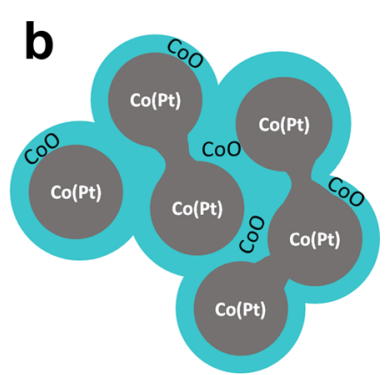

Treated at $\Delta \mathbf{V}<0$
Figure 6. Schematic drawing of (a) as-prepared state $(0 \mathrm{~V})$ and (b) treated at $-\Delta V$ to illustrate that upon application of the negative voltage, $\mathrm{Co}(\mathrm{Pt})$ metallic regions tend to grow (and eventually become more interconnected) at the expense of $\mathrm{CoO}$.

coercivity since $H_{\mathrm{C}}$ is inversely proportional to the volume of the magnetic material above the critical single-domain size. ${ }^{61}$ For isolated and isotropic Co particles, the formation of domain walls was reported above an average size of $55 \mathrm{~nm}$ (a bit larger critical sizes were reported for elongated particles). ${ }^{62}$ Although here the geometry of the alloy microdisks is much more complex than in the case of isolated particles, the interconnection and growth of the metallic counterparts within the three-dimensional nanoporous frameworks are likely to also cause a decrease of $H_{C}$.

Additionally, dipolar interactions among the different Corich clusters (which also cause a reduction of $\left.H_{\mathrm{C}}\right)^{63,64}$ are less pronounced in the as-prepared films since $\mathrm{CoO}$ tends to isolate the different magnetic zones. As the thickness of the $\mathrm{CoO}$ regions decreases, the average distance between the ferromagnetic clusters decreases (see Figure 6b) and, consequently, $H_{\mathrm{C}}$ lowers due to the enhancement of dipolar interactions. Finally, the reduction of the amount of oxygen in the Co-Pt alloy should also decrease its orbital moment (usually linked with the anisotropy), hence also contributing to the decrease in $\mathrm{H}_{\mathrm{C}}{ }^{44}$

\section{CONCLUSIONS}

In summary, large electric-field effects have been observed in nanoporous $\mathrm{Co}-\mathrm{Pt} / \mathrm{CoO}$ microdisks prepared by micelleassisted electrodeposition using optically lithographed substrates. The micrometer size of the disks and their nanoporous structure play an essential role in the magnetoelectric effect due to the resulting large surface-area-to-volume ratio. The presence of $\mathrm{CoO}$ allows for oxygen migration under the influence of the electric field. This combination allows for an unprecedented voltage control of magnetism, which is very appealing for energy-efficient magnetic actuation. Indeed, drastic changes in coercivity and the Kerr signal amplitude at 
saturation are observed at room temperature by subjecting the $\mathrm{Co}-\mathrm{Pt} / \mathrm{CoO}$ disks to a negative applied voltage using an anhydrous electrolyte. Such variations are attributed to the effective electric charge accumulation at the surface of the ultrathin pore walls and the concomitant magneto-ionic effect, which results in partial reduction from $\mathrm{CoO}$ to $\mathrm{Co}$. The reported approach can be extrapolated to other magnetic systems to foster electric-field control of magnetism in technologically relevant applications such as spintronics and magnetic actuators.

\section{EXPERIMENTAL SECTION}

4.1. Materials. Hydrochloric acid (HCL, $37 \mathrm{wt} \%)$, isopropyl alcohol $\left(\mathrm{C}_{3} \mathrm{H}_{8} \mathrm{O}, 99.9 \%\right)$, acetone $(99.0 \%)$, absolute ethanol $(99.8 \%)$, $\mathrm{Na}_{2} \mathrm{PtCl}_{6} \cdot 6 \mathrm{H}_{2} \mathrm{O}$ (sodium hexacholoroplatinate(IV) hexahydrate, $98.0 \%), \mathrm{CoCl}_{2}$ (cobalt(II) chloride anhydrous, $\geq 98.0 \%$ ), and Pluronic P-123 ( $\mathrm{HO}\left(\mathrm{CH}_{2} \mathrm{CH}_{2} \mathrm{O}\right)_{20}\left(\mathrm{CH}_{2} \mathrm{CH}\left(\mathrm{CH}_{3}\right)\right.$ $\left.\mathrm{O})_{70}\left(\mathrm{CH}_{2} \mathrm{CH}_{2} \mathrm{O}\right)_{20} \mathrm{H}\right)$ block copolymer were purchased from Sigma-Aldrich. All the reagents were used as received without further purification. AZ-9260 Photoresist and AZ-400 K Developer were purchased from Merck Performance Materials GmbH. Deionized water was obtained through an EMD Millipore Simplicity Water Purification System (Millipore S.A.S., Molsheim 67120, France).

4.2. Nanoporous $\mathrm{Co}-\mathrm{Pt} / \mathrm{CoO}$ Microdisk Growth. Electrodeposition of $\mathrm{Co}-\mathrm{Pt}$ was performed on prelithographed $\mathrm{Cu}(70 \mathrm{~nm}) /$ $\mathrm{Ti}(10 \mathrm{~nm}) / \mathrm{Si}$ substrates. Prior to patterning, the substrates were degreased with acetone, isopropanol, and Milli- $\mathrm{Q}$ water in consecutive steps. Arrays of cylindrical holes of $50 \mu \mathrm{m}$ in diameter and $5 \mu \mathrm{m}$ in height were patterned by optical lithography using photoresist AZ9260 and AZ-400 developers. Subsequently, the patterned areas were used as templates for the electrodeposition of $\mathrm{Co}-\mathrm{Pt}$ microdisks. Electrodeposition was performed in a three-electrode single-compartment cell connected to a PGSTAT302N Autolab potentiostat/ galvanostat (Methrohm-Autolab). A Pt spiral served as the counter electrode, and an $\mathrm{Ag} \mid \mathrm{AgCl}$ double junction $(E=+0.210 \mathrm{~V}$ standard hydrogen electrode), with $3 \mathrm{M} \mathrm{KCl}$ inner solution and $1 \mathrm{M} \mathrm{NaCl}$ outer solution, was employed as the reference electrode (Figure 1a). The electrolyte was prepared with Milli- $\mathrm{Q}$ water and contained 1.3 $\mathrm{mM} \mathrm{Na} \mathrm{PtCl}_{6} \cdot 6 \mathrm{H}_{2} \mathrm{O}, 2.8 \mathrm{mM} \mathrm{CoCl}$, and $1 \mathrm{mg} / \mathrm{mL}$ (1 wt \%) of Pluronic $\mathrm{P}-123$. The $\mathrm{pH}$ was adjusted to 2.1 with $\mathrm{HCl}$ solution. The block-copolymer P-123 was used above its critical micellar concentration $^{65}$ to ensure the formation of micelles in the aqueous electrolyte. These micelles interact with the metal ions in solution, and once adsorbed on the patterned holes of the working electrode, the discharge of the cations results in the formation of the nanoporous film (Figure 1a). Importantly, the electrolyte did not contain a $\mathrm{pH}$ buffering agent so as to favor the growth of a nanocomposite coating consisting of a Co-rich alloy and Co oxides. In the absence of $\mathrm{pH}$ buffering agents, solution alkalinization at the cathode causes hydroxide/oxide precipitation. Therefore, oxygen can be incorporated in the film, forming oxides. Electrodeposition was conducted potentiostatically at $-1.0 \mathrm{~V}$ for $350 \mathrm{~s}$, under mild stirring $(\omega=100$ rpm) and simultaneous bubbling of $\mathrm{N}_{2}$ through the solution. The temperature of the electrolyte was kept at $25^{\circ} \mathrm{C}$ by circulating water through the outer jacket of the electrolyte cell using an F12 Julabo thermostat. A representative current-time curve is shown in the Supporting Information (Figure S5). Finally, the photoresist was removed by immersing the samples in acetone, followed by a final rising in isopropanol and Milli-Q water.

4.3. Morphology and Structural Characterization. The morphology of the nanoporous $\mathrm{Co}-\mathrm{Pt}$ microdisks was examined by field emission scanning electron microscopy (FE-SEM) using a Zeiss MERLIN operated at $5 \mathrm{kV}$. Compositional analysis was performed by energy-dispersive X-ray spectroscopy (EDX) using the FE-SEM operated at $15 \mathrm{kV}$. The crystal structure was investigated by means of high-resolution transmission electron microscopy (HRTEM) and selected area electron diffraction (SAED) utilizing a JEOL-JEM 2011 operated at $200 \mathrm{kV}$. Further morphological and compositional characterization along the cross section of a microdisk was carried out by electron energy loss spectroscopy (EELS) analysis on a Tecnai F20 HRTEM/STEM microscope. TEM, STEM, and EELS analyses were performed on the cross section of the disks, from a lamella prepared by focused ion beam (FIB) (Crossbeam 1560XB from Zeiss with an ion column Canion from Orsay Physics). To prepare the lamella, the nanoporous film was capped with a $2 \mu$ m-thick TEOS layer, and a slice of material with dimensions of approximately $1 \times 10$ $\mu \mathrm{m}^{2}$ was then cut and glued onto a TEM grid to be further thinned down to $100 \mathrm{~nm}$ using $5 \mathrm{kV}$ and a low current $(50 \mathrm{pA})$. To assess the influence of voltage on the microstructure of the nanoporous films, EELS analyses were performed before and after subjecting the lamella to $-14 \mathrm{~V}$ for $40 \mathrm{~min}$ in the same electrolyte used for the magnetoelectric measurements.

4.4. Soft X-ray Absorption Spectroscopy Characterization. The chemical composition and oxidation state of the nanoporous $\mathrm{Co}-\mathrm{Pt}$ microdisks were determined by soft $\mathrm{X}$-ray absorption spectra (XAS) ( $\mathrm{Co}_{3,2}$ edge), measured in total electron yield mode and using linearly polarized light, in the beamline BL24-CIRCE at the ALBA Synchrotron. ${ }^{66}$ The XAS patterns of reference Co $(20 \mathrm{~nm}$-thick Co thin film, capped with $2 \mathrm{~nm}$ of $\mathrm{Ta}$, grown by molecular beam epitaxy on top of a $\langle 100\rangle$-oriented $\mathrm{MgO}$ single crystal) and $\mathrm{CoO}$ (cobalt(II) oxide, 95\%, powders from Alfa Aesar) samples were also acquired for comparison.

4.5. Magnetoelectric Measurements. In-plane hysteresis loops at different values of applied DC voltage were acquired at room temperature in situ in a magneto-optical Kerr effect (MOKE) setup from Durham Magneto-Optics. An Agilent B2902A power supply was employed as the voltage source. The sample was mounted vertically on a poly (methyl methacrylate) holder placed in a quartz SUPRASIL cell filled with anhydrous propylene carbonate containing $\mathrm{Na}^{+}$and $\mathrm{OH}^{-}$ions. The anhydricity of the electrolyte minimized the occurrence of extrinsic oxidation or corrosion events in the $\mathrm{Co}-\mathrm{Pt}$ microdisks during magnetoelectric measurements. To remove any traces of residual water in the electrolyte, this was treated with metallic sodium. To prepare the electrolyte, a piece of metallic sodium was immersed in a flask containing PC (the metallic $\mathrm{Na}$ was in excess to the water molecules present in the PC), which was then stored in a glovebox. To be able to perform the magnetoelectric measurements, the quartz cell was filled in with the electrolyte using a syringe. Between each increase or decrease of the applied voltage, a minimum waiting time of $300 \mathrm{~s}$ was taken before the hysteresis loop was measured (in situ) to enable the electrolyte to diffuse through the nanopores and permit the formation of the electrical double layer within the entire 3D network.

\section{ASSOCIATED CONTENT}

\section{Supporting Information}

The Supporting Information is available free of charge on the ACS Publications website at DOI: 10.1021/acsami.8b17442.

EDX data, electron diffraction data, MOKE measurements, cyclic voltammetry experiments and electrodeposition curve (PDF)

\section{AUTHOR INFORMATION}

\section{Corresponding Authors}

*E-mail: Cristina.navarro@e-campus.uab.cat (C.N.-S.).

*E-mail: Eva.Pellicer@uab.cat (E.P.).

*E-mail: Jordi.Sort@uab.cat (J.S.).

ORCID $\odot$

Alberto Quintana: 0000-0002-9813-735X

Eva Pellicer: 0000-0002-8901-0998

Jordi Sort: 0000-0003-1213-3639 


\section{Author Contributions}

The manuscript was written through contributions of all authors. All authors have given approval to the final version of the manuscript.

\section{Notes}

The authors declare no competing financial interest.

\section{ACKNOWLEDGMENTS}

This work was funded by the European Research Council under the SPIN-PORICS 2014-Consolidator Grant (Agreement No. 648454), the Generalitat de Catalunya (2017-SGR292 project), the Spanish Government (MAT2017-86357-C31-R, MAT2014-57960-C3-1-R, and associated FEDER projects), and the European Union's Horizon 2020 research and innovation program under the Marie Skłodowska-Curie grant agreement No. 665919. J.F. and E.P. acknowledge the Juan de la Cierva (IJCI-2015-27030) and Ramon y Cajal (RYC-201210839) fellowships, respectively, from MINECO. The ICN2 is funded by the CERCA program/Generalitat de Catalunya. ICN2 also acknowledges the support from the Severo Ochoa Program (MINECO, grant SEV-2013-0295).

\section{REFERENCES}

(1) Žutić, I.; Fabian, J.; Sarma, S. D. Spintronics: Fundamentals and Applications. Rev. Mod. Phys. 2004, 76, 323-410.

(2) Wolf, S. A. Spintronics: A Spin-Based Electronics Vision for the Future. Science 2001, 294, 1488-1495.

(3) Chappert, C.; Fert, A.; Dau, F. N. V. The Emergence of Spin Electronics in Data Storage. Nat. Mater. 2007, 6, 813-823.

(4) Dieny, B.; Sousa, R.; Herault, J.; Papusoi, C.; Prenat, G.; Ebels, U.; Houssameddine, D.; Rodmacq, B.; Auffret, S.; Prejbeanu, L. B.; Cyrille, M.; Delaet, B.; Redon, O.; Ducruet, C.; Nozieres, J. P.; Prejbeanu, I. Spin-Transfer Effect and Its Use in Spintronic Components. Int. J. Nanotechnol. 2010, 7, 591-614.

(5) Hu, J.-M.; Li, Z.; Chen, L.-Q.; Nan, C.-W. High-Density Magnetoresistive Random Access Memory Operating at Ultralow Voltage at Room Temperature. Nat. Commun. 2011, 2, No. 553.

(6) Brovko, O. O.; Ruiz-Díaz, P.; Dasa, T. R.; Stepanyuk, V. S. Controlling Magnetism on Metal Surfaces with Non-Magnetic Means: Electric Fields and Surface Charging. J. Phys.: Condens. Matter 2014, 26, No. 093001.

(7) Song, C.; Cui, B.; Li, F.; Zhou, X.; Pan, F. Recent Progress in Voltage Control of Magnetism: Materials, Mechanisms, and Performance. Prog. Mater. Sci. 2017, 87, 33-82.

(8) Duschek, K.; Pohl, D.; Fähler, S.; Nielsch, K.; Leistner, K. Research Update: Magnetoionic Control of Magnetization and Anisotropy in Layered Oxide/Metal Heterostructures. APL Mater. 2016, 4, No. 032301.

(9) Eerenstein, W.; Mathur, N. D.; Scott, J. F. Multiferroic and Magnetoelectric Materials. Nature 2006, 442, 759-765.

(10) Wang, Y.; Hu, J.; Lin, Y.; Nan, C.-W. Multiferroic Magnetoelectric Composite Nanostructures. NPG Asia Mater. 2010, 2, 61-68. (11) Lu, C.; Hu, W.; Tian, Y.; Wu, T. Multiferroic Oxide Thin Films and Heterostructures. Appl. Phys. Rev. 2015, 2, No. 021304.

(12) Brivio, S.; Petti, D.; Bertacco, R.; Cezar, J. C. Electric Field Control of Magnetic Anisotropies and Magnetic Coercivity in $\mathrm{Fe} /$ $\mathrm{BaTiO}_{3}(001)$ Heterostructures. Appl. Phys. Lett. 2011, 98, No. 092505.

(13) Liu, Y.; Hu, F.-X.; Zhang, M.; Wang, J.; Shen, F.-R.; Zuo, W.-L.; Zhang, J.; Sun, J.-R.; Shen, B.-G. Electric Field Control of Magnetic Properties of $\mathrm{Nd}_{2} \mathrm{Fe}_{14} \mathrm{~B}$ Thin Films Grown onto PMN-PT Substrates. Appl. Phys. Lett. 2017, 110, No. 022401.

(14) Fina, I.; Quintana, A.; Padilla-Pantoja, J.; Martí, X.; Macià, F.; Sánchez, F.; Foerster, M.; Aballe, L.; Fontcuberta, J.; Sort, J. ElectricField-Adjustable Time-Dependent Magnetoelectric Response in
Martensitic FeRh Alloy. ACS Appl. Mater. Interfaces 2017, 9, 15577-15582.

(15) Weisheit, M.; Fahler, S.; Marty, A.; Souche, Y.; Poinsignon, C.; Givord, D. Electric Field-Induced Modification of Magnetism in Thin-Film Ferromagnets. Science 2007, 315, 349-351.

(16) Koyama, T.; Chiba, D. Influence of the Magnetization Reversal Mechanism on the Electric Field Modulation of Coercivity in Pt/Co Structures. Phys. Rev. B 2017, 96, No. 224409.

(17) Chiba, D.; Ono, T. Control of Magnetism in Co by an Electric Field. J. Phys. D: Appl. Phys. 2013, 46, No. 213001.

(18) Chiba, D.; Fukami, S.; Shimamura, K.; Ishiwata, N.; Kobayashi, K.; Ono, T. Electrical Control of the Ferromagnetic Phase Transition in Cobalt at Room Temperature. Nat. Mater. 2011, 10, 853-856.

(19) Maruyama, T.; Shiota, Y.; Nozaki, T.; Ohta, K.; Toda, N.; Mizuguchi, M.; Tulapurkar, A. A.; Shinjo, T.; Shiraishi, M.; Mizukami, S.; Ando, Y.; Suzuki, Y. Large Voltage-Induced Magnetic Anisotropy Change in a Few Atomic Layers of Iron. Nat. Nanotechnol. 2009, 4, $158-161$.

(20) Zhang, H.; Richter, M.; Koepernik, K.; Opahle, I.; Tasnádi, F.; Eschrig, H. Electric-Field Control of Surface Magnetic Anisotropy: A Density Functional Approach. New J. Phys. 2009, 11, No. 043007.

(21) Yang, Q.; Wang, L.; Zhou, Z.; Wang, L.; Zhang, Y.; Zhao, S.; Dong, G.; Cheng, Y.; Min, T.; Hu, Z.; Chen, W.; Xia, K.; Liu, M. Ionic Liquid Gating Control of RKKY Interaction in $\mathrm{FeCoB} / \mathrm{Ru} /$ $\mathrm{FeCoB}$ and $(\mathrm{Pt} / \mathrm{Co})_{2} / \mathrm{Ru} /(\mathrm{Co} / \mathrm{Pt})_{2}$ Multilayers. Nat. Commun. 2018, 9, No. 991.

(22) Ohno, H.; Chiba, D.; Matsukura, F.; Omiya, T.; Abe, E.; Dietl, T.; Ohno, Y.; Ohtani, K. Electric-Field Control of Ferromagnetism. Nature 2000, 408, 944-946.

(23) Chiba, D.; Matsukura, F.; Ohno, H. Electrically Defined Ferromagnetic Nanodots. Nano Lett. 2010, 10, 4505-4508.

(24) Jiang, S.; Shan, J.; Mak, K. F. Electric-Field Switching of TwoDimensional Van Der Waals Magnets. Nat. Mater. 2018, 17, 406410

(25) Bauer, U.; Emori, S.; Beach, G. S. D. Voltage-Gated Modulation of Domain Wall Creep Dynamics in an Ultrathin Metallic Ferromagnet. Appl. Phys. Lett. 2012, 101, No. 172403.

(26) Bauer, U.; Yao, L.; Tan, A. J.; Agrawal, P.; Emori, S.; Tuller, H. L.; Dijken, S. V.; Beach, G. S. D. Magneto-Ionic Control of Interfacial Magnetism. Nat. Mater. 2015, 14, 174-181.

(27) Gilbert, D. A.; Grutter, A. J.; Arenholz, E.; Liu, K.; Kirby, B. J.; Borchers, J. A.; Maranville, B. B. Structural and Magnetic Depth Profiles of Magneto-Ionic Heterostructures beyond the Interface Limit. Nat. Commun. 2016, 7, No. 12264.

(28) Baldrati, L.; Tan, A. J.; Mann, M.; Bertacco, R.; Beach, G. S. D. Magneto-Ionic Effect in $\mathrm{CoFeB}$ Thin Films with in-Plane and Perpendicular-to-Plane Magnetic Anisotropy. Appl. Phys. Lett. 2017, 110, No. 012404

(29) Bi, C.; Liu, Y.; Newhouse-Illige, T.; Xu, M.; Rosales, M.; Freeland, J. W.; Mryasov, O.; Zhang, S.; Velthuis, S. G. E. T.; Wang, W. G. Reversible Control of Co Magnetism by Voltage-Induced Oxidation. Phys. Rev. Lett. 2014, 113, No. 267202.

(30) Duschek, K.; Petr, A.; Zehner, J.; Nielsch, K.; Leistner, K. AllElectrochemical Voltage-Control of Magnetization in Metal Oxide/ Metal Nanoislands. J. Mater. Chem. C 2018, 6, 8411-8417.

(31) Porro, S.; Betjka, K.; Jasmin, A.; Fontana, M.; Milano, G.; Chiolerio, A.; Fabrizio Pirri, C.; Ricciardi, C. A Multi-Level Memristor Based on Atomic Layer Deposition of Iron Oxide. Nanotechnology 2018, 29, No. 495201.

(32) Black, C.; Welser, J. Electric-Field Penetration into Metals: Consequences for High-Dielectric-Constant Capacitors. IEEE Trans. Electron Devices 1999, 46, 776-780.

(33) Schmickler, W. Electronic Effects in the Electric Double Layer. Chem. Rev. 1996, 96, 3177-3200.

(34) Quintana, A.; Zhang, J.; Isarain-Chávez, E.; Menéndez, E.; Cuadrado, R.; Robles, R.; Baró, M. D.; Guerrero, M.; Pané, S.; Nelson, B. J.; Müller, C. M.; Ordejón, P.; Nogués, J.; Pellicer, E.; Sort, J. Voltage-Induced Coercivity Reduction in Nanoporous Alloy Films: 
A Boost toward Energy-Efficient Magnetic Actuation. Adv. Funct. Mater. 2017, 27, No. 1701904.

(35) Dislaki, E.; Robbennolt, S.; Campoy-Quiles, M.; Nogués, J.; Pellicer, E.; Sort, J. Coercivity Modulation in $\mathrm{Fe}-\mathrm{Cu}$ Pseudo-Ordered Porous Thin Films Controlled by an Applied Voltage: A Sustainable, Energy-Efficient Approach to Magnetoelectrically Driven Materials. Adv. Sci. 2018, 5, No. 1800499.

(36) Yamada, Y.; Ueno, K.; Fukumura, T.; Yuan, H. T.; Shimotani, H.; Iwasa, Y.; Gu, L.; Tsukimoto, S.; Ikuhara, Y.; Kawasaki, M. Electrically Induced Ferromagnetism at Room Temperature in Cobalt-Doped Titanium Dioxide. Science 2011, 332, 1065-1067.

(37) Dasgupta, S.; Das, B.; Li, Q.; Wang, D.; Baby, T. T.; Indris, S.; Knapp, M.; Ehrenberg, H.; Fink, K.; Kruk, R.; Hahn, H. Toward Onand-Off Magnetism: Reversible Electrochemistry to Control Magnetic Phase Transitions in Spinel Ferrites. Adv. Funct. Mater. 2016, 26, $7507-7515$.

(38) Zhou, X.; Yan, Y.; Jiang, M.; Cui, B.; Pan, F.; Song, C. Role of Oxygen Ion Migration in the Electrical Control of Magnetism in Pt/ Co/Ni/HfO2 Films. J. Phys. Chem. C 2016, 120, 1633-1639.

(39) Shimatsu, T.; Sato, H.; Oikawa, T.; Inaba, Y.; Kitakami, O.; Okamoto, S.; Aoi, H.; Muraoka, H.; Nakamura, Y. High Perpendicular Magnetic Anisotropy of $\mathrm{CoPtCr} / \mathrm{Ru}$ Films for Granular-Type Perpendicular Media. IEEE Trans. Magn. 2004, 40, 2483-2485.

(40) Zana, I.; Zangari, G. Electrodeposition of Co-Pt Films with High Perpendicular Anisotropy. Electrochem. Solid State Lett. 2003, 6, C153-C156.

(41) Rožman, K. Ž.; Krause, A.; Leistner, K.; Fähler, S.; Schultz, L.; Schlörb, H. Electrodeposition and Hard Magnetic Properties of CoPt Films in Comparison to Fe-Pt Films. J. Magn. Magn. Mater. 2007, $314,116-121$.

(42) Cortés, M.; Serrà, A.; Gómez, E.; Vallés, E. CoPt Nanoscale Structures with Different Geometry Prepared by Electrodeposition for Modulation of Their Magnetic Properties. Electrochim. Acta 2011, 56, $8232-8238$.

(43) Reichel, L.; Oswald, S.; Fähler, S.; Schultz, L.; Leistner, K. Electrochemically Driven Variation of Magnetic Properties in Ultrathin CoPt Films. J. Appl. Phys. 2013, 113, No. 143904.

(44) Imperia, P.; Glaser, L.; Martins, M.; Andreazza, P.; Penuelas, J.; Alessandrovic, V.; Weller, H.; Andreazza-Vignolle, C.; Wurth, W. XMCD Studies of $\mathrm{Co}_{\mathrm{x}} \mathrm{Pt}_{100-\mathrm{X}}$ nanoparticles Prepared by Vapour Deposition and Chemical Synthesis. Phys. Status Solidi A 2008, 205, 1047-1051.

(45) Yamauchi, Y.; Tonegawa, A.; Komatsu, M.; Wang, H.; Wang, L.; Nemoto, Y.; Suzuki, N.; Kuroda, K. Electrochemical Synthesis of Mesoporous Pt-Au Binary Alloys with Tunable Compositions for Enhancement of Electrochemical Performance. J. Am. Chem. Soc. 2012, 134, 5100-5109.

(46) Isarain-Chávez, E.; Baró, M. D.; Pellicer, E.; Sort, J. MicelleAssisted Electrodeposition of Highly Mesoporous Fe-Pt Nodular Films with Soft Magnetic and Electrocatalytic Properties. Nanoscale 2017, 9, 18081-18093.

(47) Pellicer, E.; Pané, S.; Sivaraman, K.; Ergeneman, O.; Suriñach, S.; Baró, M.; Nelson, B.; Sort, J. Effects of the Anion in GlycineContaining Electrolytes on the Mechanical Properties of Electrodeposited Co-Ni Films. Mater. Chem. Phys. 2011, 130, 1380-1386. (48) Pattanaik, G.; Zangari, G. Morphology and Magnetic Properties of Co-Rich Co-Pt Thin Films Electrodeposited on Cr Seed Layers. J. Electrochem. Soc. 2006, 153, C6-C10.

(49) Tang, K.; Fu, L.; White, R. J.; Yu, L.; Titirici, M.-M.; Antonietti, M.; Maier, J. Hollow Carbon Nanospheres with Superior Rate Capability for Sodium-Based Batteries. Adv. Energy Mater. 2012, 2, 873-877.

(50) Yan, Y.; Yin, Y.-X.; Guo, Y.-G.; Wan, L.-J. A Sandwich-Like Hierarchically Porous Carbon/Graphene Composite as a HighPerformance Anode Material for Sodium-Ion Batteries. Adv. Energy Mater. 2014, 4, No. 1301584.

(51) Robbennolt, S.; Quintana, A.; Pellicer, E.; Sort, J. Large Magnetoelectric Effects Mediated by Electric-Field-Driven Nanoscale Phase Transformations in Sputtered (Nanoparticulate) and Electro- chemically Dealloyed (Nanoporous) Fe-Cu Films. Nanoscale 2018, $10,14570-14578$.

(52) Quintana, A.; Menéndez, E.; Liedke, M. O.; Butterling, M.; Wagner, A.; Sireus, V.; Torruella, P.; Estradé, S.; Peiró, F.; Dendooven, J.; Detavernier, C.; Murray, P. D.; Gilbert, D. A.; Liu, K.; Pellicer, E.; Nogues, J.; Sort, J. Voltage-Controlled ON-OFF Ferromagnetism at Room Temperature in a Single Metal Oxide Film. ACS Nano 2018, 12, 10291-10300.

(53) Dasa, T. R.; Ignatiev, P. A.; Stepanyuk, V. S. Effect of the Electric Field on Magnetic Properties of Linear Chains on a $\mathrm{Pt}(111)$ Surface. Phys. Rev. B 2012, 85, No. 205447.

(54) Topolovec, S.; Jerabek, P.; Szabó, D. V.; Krenn, H.; Würschum, R. SQUID Magnetometry Combined with in Situ Cyclic Voltammetry: A Case Study of Tunable Magnetism of $\gamma-\mathrm{Fe}_{2} \mathrm{O}_{3}$ Nanoparticles. J. Magn. Magn. Mater. 2013, 329, 43-48.

(55) Subkow, S.; Fähnle, M. Potential Explanation of Charge Response of Magnetization in Nanoporous Systems. Phys. Rev. B 2011, 84, No. 220409.

(56) Drings, H.; Viswanath, R. N.; Kramer, D.; Lemier, C.; Weissmüller, J.; Würschum, R. Tuneable Magnetic Susceptibility of Nanocrystalline Palladium. Appl. Phys. Lett. 2006, 88, No. 253103.

(57) Ghosh, S. Charge-Response of Magnetization in Nanoporous Pd-Ni Alloys. J. Magn. Magn. Mater. 2011, 323, 552-556.

(58) Regan, T. J.; Ohldag, H.; Stamm, C.; Nolting, F.; Lüning, J.; Stöhr, J.; White, R. L. Chemical Effects at Metal/Oxide Interfaces Studied by x-Ray-Absorption Spectroscopy. Phys. Rev. B 2001, 64, No. 214422.

(59) Menéndez, E.; Modarresi, H.; Dias, T.; Geshev, J.; Pereira, L. M. C.; Temst, K.; Vantomme, A. Tuning the FerromagneticAntiferromagnetic Interfaces of Granular $\mathrm{Co}-\mathrm{CoO}$ Exchange Bias Systems by Annealing. J. Appl. Phys. 2014, 115, No. 133915.

(60) Menéndez, E.; Demeter, J.; Eyken, J. V.; Nawrocki, P.; Jedryka, E.; Wójcik, M.; Lopez-Barbera, J. F.; Nogués, J.; Vantomme, A.; Temst, K. Improving the Magnetic Properties of $\mathrm{Co}-\mathrm{CoO}$ Systems by Designed Oxygen Implantation Profiles. ACS Appl. Mater. Interfaces 2013, 5, 4320-4327.

(61) Hadjipanayis, G. C. Nanophase Hard Magnets. J. Magn. Magn. Mater. 1999, 200, 373-391.

(62) Sato, M.; Ishii, Y. Critical Sizes of Cobalt Fine Particles with Uniaxial Magnetic Anisotropy. J. Appl. Phys. 1983, 54, 1018-1020.

(63) Kechrakos, D.; Trohidou, K. N. Magnetic Properties of Dipolar Interacting Single-Domain Particles. Phys. Rev. B 1998, 58, 1216912177.

(64) Skomski, R.; Coey, J. M. D. Permanent Magnetism; Institute of Physics Publishing: Bristol, 1999.

(65) Wanka, G.; Hoffmann, H.; Ulbricht, W. Phase Diagrams and Aggregation Behavior of Poly(Oxyethylene)-Poly(Oxypropylene)Poly(Oxyethylene) Triblock Copolymers in Aqueous Solutions. Macromolecules 1994, 27, 4145-4159.

(66) Aballe, L.; Foerster, M.; Pellegrin, E.; Nicolas, J.; Ferrer, S. The ALBA Spectroscopic LEEM-PEEM Experimental Station: Layout and Performance. J. Synchrotron Radiat. 2015, 22, 745-752. 